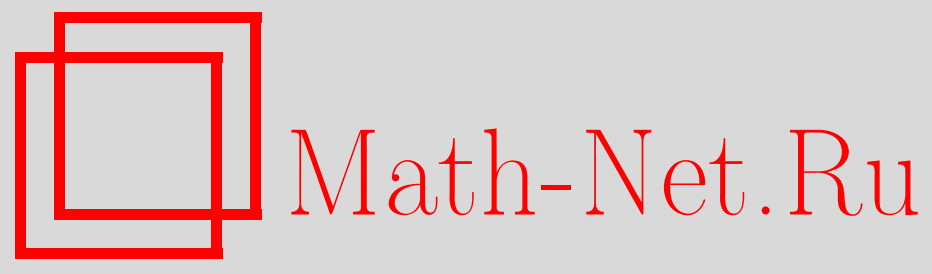

Ф. С. Насыров, Симметричные интегралы и стохастический анализ, Теория вероятн. и ее примен., 2006, том 51, выпуск 3, 496-517

DOI: https://doi.org/10.4213/tvp36

Использование Общероссийского математического портала MathNet.Ru подразумевает, что вы прочитали и согласны с пользовательским соглашением

http://www . mathnet.ru/rus/agreement

Параметры загрузки:

IP : 54.197 .130 .99

26 апреля 2023 г., 12:57:58

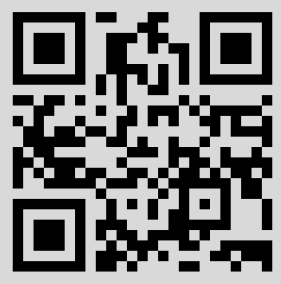




\title{
СИММЕТРИЧНЫЕ ИНТЕГРАЛЫ И СТОХАСТИЧЕСКИЙ АНАЛИЗ ${ }^{1)}$
}

\begin{abstract}
Определяются симметричные интегралы типа Стилтьеса по произвольной непрерывной функции, которые являются детерминированными версиями стохастических интегралов Стратоновича. Показано, что решение потраекторных аналогов стохастических дифференциальных уравнений с симметричным интегралом и стохастических дифференциальных уравнений Ито удается свести к решению системы обыкновенных дифференциальных уравнений первого порядка. Устанавливается связь между несобственными симметричными интегралами, расширенными симметричными интегралами и интегралами Хеллингера.

Ключевые слова и фразы: симметричный интеграл, стохастический интеграл Стратоновича, стохастические дифференциальные уравнения, несобственный симметричный интеграл, расширенный симметричный интеграл, интеграл Хеллингера.
\end{abstract}

1. Введение. Тематика статьи восходит к работе Л. Янга [12], где впервые были построены интегралы типа Стилтьеса по функциям неограниченной вариации. В работах [2], [3], [7], [11], [12] исследованы различные варианты интегралов такого вида, в частности, интегралы Хеллингера. В то же время в стохастическом анализе хорошо известны стохастические интегралы Стратоновича, которые не имели своего «потраекторного» аналога. В работе автора [6] для определенного класса интеграндов построены симметричные интегралы по произвольной непрерывной функции, которые являются детерминированными вариантами стохастических интегралов Стратоновича; при этом никаких предположений о предсказуемости интеграндов при потраекторном подходе не требуется.

Настоящую статью можно считать продолжением работы [6]. В п. 2 приводятся условия, при которых симметричный интеграл по любой непрерывной функции сушествует, и доказывается корректность опреде-

* Уфимский государственный авиационный технический университет, кафедра математики, ул. К. Маркса, 12, 450000 Уфа, Россия; e-mail: farsagit@yandex.ru

1) Работа выполнена при финансовой поддержке РФФИ (гранты 04-01-00286a, 05-01-97909). 
ления симметричного интеграла. В п. 3 рассматриваются детерминированные аналоги стохастических дифференциальных уравнений с симметричными интегралами. С точки зрения теории случайных процессов коэффициенты рассматриваемых уравнений могут быть как детерминированными, так и случайными и не обязаны быть предсказуемыми функциями.

Показано, что при определенных условиях, накладываемых на коэффициенты, решение такого вида уравнений может быть сведено к решению системы из двух обыкновенных дифференциальных уравнений первого порядка. Кроме того, с помощью подхода А. В. Скорохода удалось построить детерминированные аналоги стохастических дифференциальных уравнений с отражающим экраном. Решение стохастических дифференциальных уравнений со стохастическим интегралом Ито в случае гладких коэффициентов также сводится к решению системы обыкновенных дифференциальных уравнений первого порядка.

В п. 4 устанавливается связь между несобственными симметричными интегралами и другими известными конструкциями интегралов по функциям неограниченной вариации. Доказывается, что расширенный симметричный интеграл (см. [6]), являющийся интегралом по определенного вида заряду, для некоторого класса интеграндов является пределом симметричньх интегралов, т.е. является несобственным симметричным интегралом. Выяснилось, что другой класс несобственных симметричных интегралов связан с интегралами Хеллингера.

Таким образом, основным результатом данной статьи можно считать тот факт, что методами теории функций оказалось возможным построить детерминированный аналог стохастического исчисления Стратоновича; естественно, это «параллельное» исчисление не может охватить всю красоту стохастического исчисления, однако оно показывает, что часть результатов, ранее справедливых в рамках теории мартингалов, имеет более общую природу.

Приведем необходимые обозначения и сведения. Множества $\mathbf{R}=$ $(-\infty,+\infty)$ и $[0, t], t>0$, предполагаются наделенными $\sigma$-алгебрами борелевских множеств, которые обозначаются соответственно $\mathscr{B}$ и $\mathscr{B} t, t>0$; на этих подмножествах считается заданной мера Лебега $\lambda(\cdot)$. Для непрерывной функции $X(s), s \in[0,+\infty)$, положим

$$
\begin{aligned}
& M(t)=\max \{X(s): s \in[0, t]\}, \quad m(t)=\min \{X(s): s \in[0, t]\}, \\
& M^{-1}(u)=\inf \{s: M(s)>u\}, \quad m^{-1}(u)=\inf \{s: m(s)<u\} .
\end{aligned}
$$

Обозначим $\operatorname{sign}(x)$ знак вещественного числа $x$, а $1(A)$ будет обозначать индикатор множества $A$, т.е. функцию, равную 1 на $A$ и 0 вне $A$; далее всюду $a \wedge b=\min (a, b), a \vee b=\max (a, b), \kappa(v, a, b)=\operatorname{sign}(b-a) \mathbf{1}(a \wedge b<$ $v<a \vee b)$. 
Пусть $X(s), s \in[0,1],-$ борелевская вещественнозначная функция, $\tau(\cdot)$ - мера на $\sigma$-алгебре $\mathscr{B}_{1}$ борелевских множеств отрезка $[0,1]$. Введем меры

$$
\nu_{t}(\Gamma)=\int_{0}^{t} \mathbf{1}(X(s) \in \Gamma) \tau(d s), \quad \Gamma \in \mathscr{B}, \quad t \in[0,1],
$$

тогда $\nu_{t}(\Gamma)$ есть «количество времени», проводимое функцией $X(s), s \in$ $[0, t]$, в множестве $\Gamma$. Производная Радона-Никодима

$$
\alpha_{\tau}(t, u) \equiv \alpha_{t}(u)=\frac{d \nu_{t}}{d \lambda}(u), \quad u \in \mathbf{R},
$$

если она существует, называется локальным временем функции $X(s)$. Оказывается (см. [10]), можно всегда считать, что локальное время измеримо как функция двух переменных и является при каждом $u$ неубывающей непрерывной справа функцией по $t$; меру на $\mathscr{B}_{1}$, которую она порождает, мы будем обозначать $\alpha_{\tau}(d s, u)$.

Из определения локального времени следует (см. [10]), что для любой ограниченной (или знакопостоянной) борелевской функции $f(s, u)$ справедливо равенство

$$
\int_{0}^{t} f(s, X(s)) \tau(d s)=\int_{\mathbf{R}} \int_{0}^{t} f(s, u) \alpha(d s, u) d u .
$$

Говорят, что случайный процесс обладает локальным временем, если почти все его траектории имеют локальное время. В случае, когда $\tau(d s)=d s$, положим $\alpha_{\tau}(t, u) \equiv \alpha(t, u)$. Пусть $X(s)$ - абсолютно непрерывная функция, $\tau(d s)=\left|X^{\prime}(s)\right| d s$, тогда локальное время $N(t, u) \equiv$ $\alpha_{\tau}(t, u)$ для функции $X(s)$ называется (см. [4]) индикатрисой Банаха; величина $N(t, u)$ равна числу пересечений функцией $X(s), s \in[0, t]$, уровня $u$.

Ряд общих сведений о локальных временах детерминированных функций и случайных процессов приведен в работах [1], [5], [8]-[10].

\section{2. Симметричный интеграл и его вычисление.}

О п р е д е л е н и е. Будем говорить, что пара функций $X(s)$, $s \in[0,1]$, и $f(s, u), s \in[0,1], u \in \mathbf{R}$, удовлетворяет условию (S) на $[0, t]$, если:

(a) функция $X(s), s \in[0, t]$, непрерывна;

(b) при почти всех $u$ функция $f(s, u), s \in[0, t]$, имеет ограниченное изменение и непрерывна справа по $s \in[0, t]$;

(c) при почти всех $u$ справедливо равенство

$$
\int_{0}^{t} \mathbf{1}(X(s)=u)|f|(d s, u)=0,
$$


где при каждом $u$ функция $|f|(s, u)$ есть полное изменение функции $f(\tau, u)$ по переменной $\tau$ на отрезке $[0, s]$;

(d) полное изменение $|f|(t, u)$ функции $f(s, u)$ по переменной $s$ на отрезке $[0, t]$ локально суммируемо по $u$.

3 а м е ч а н и я. 1. Условие (c) будет выполнено, если непрерывная функция $X(s)$ обладает локальным временем $\alpha(t, u)$, а функция $f(s, u)$ при почти всех $u$ абсолютно непрерывна по переменной $s$.

2. Если $X(s)=X(s, \omega)$ - случайный процесс, обладающий локальным временем $\alpha(t, u)$, а $f(s, u)$ - детерминированная функция, удовлетворяющая условию (b), то условие (c) будет выполнено для почти всех траекторий процесса. В частности, для случайного процесса броуновского движения $X(s, \omega)$ и детерминированной функции $f(s, u)$ с условием (b) предположение (c) всегда выполняется.

Пусть функции $X(s)$ и $f(s, u)$ удовлетворяют условию $(\mathrm{S})$ на отрезке $[0, t]$. Рассмотрим разбиения $T_{n}, n \in \mathbf{N}$, отрезка $[0, t]: T_{n}=\left\{t_{k}^{(n)}\right\}$, $0=t_{0}^{(n)} \leqslant t_{1}^{(n)} \leqslant \cdots \leqslant t_{k}^{(n)} \leqslant \cdots \leqslant t_{m_{n}}^{(n)}=t, n \in \mathbf{N}$, такие, что $T_{n} \subset T_{n+1}$, $n \in \mathbf{N}$, и $\lambda_{n}=\max _{k}\left|t_{k}^{(n)}-t_{k-1}^{(n)}\right| \rightarrow 0$ при $n \rightarrow \infty$. Через $X^{(n)}(s)$, $s \in[0, t]$, обозначим ломаную, построенную по функции $X(s)$ и отвечающую разбиению $T_{n}$, а через $N^{(n)}(t, u)$ соответствуюшую ей индикатрису Банаха. Введем также следующие обозначения: $\Delta t_{k}^{(n)}=t_{k}^{(n)}-t_{k-1}^{(n)}$, $\left[\Delta t_{k}^{(n)}\right]=\left[t_{k-1}^{(n)}, t_{k}^{(n)}\right], \Delta X_{k}^{(n)}=X\left(t_{k}^{(n)}\right)-X\left(t_{k-1}^{(n)}\right)$.

О п р е д е л е и е. Симметричным интегралом называется (cM. [5])

$$
\int_{0}^{t} f(s, X(s)) * d X(s)=\lim _{n \rightarrow \infty} \sum_{k} \frac{1}{\Delta t_{k}^{(n)}} \int_{\left[\Delta t_{k}^{(n)}\right]} f\left(s, X^{(n)}(s)\right) d s \Delta X_{k}^{(n)}
$$

если предел в правой части равенства существует и не зависит от выбора последовательности разбиений $T_{n}, n \in \mathbf{N}$.

3 а м е ч а н и е. Симметричный интеграл является (см. [6]) детерминированным аналогом стохастического интеграла Стратоновича.

Лемма 1. Пусть $X(s), s \in[0,1],-$ произвольная непрерывная бункция, $X^{(n)}(s), s \in[0,1]$, ломаная, построенная по разбиению $T_{n}$. Тогда для всех $v$, которые удовлетворяют условию

$$
\int_{t_{1}}^{t_{2}} \mathbf{1}\left(X^{(n)}(s)=v\right) d s=0
$$

интеграл $\int_{t_{1}}^{t_{2}} \operatorname{sign}\left(\left(X^{(n)}\right)^{\prime}(s)\right) N^{(n)}(d s, v)$ равен $\operatorname{sign}\left(X^{(n)}\left(t_{2}\right)-X^{(n)}\left(t_{1}\right)\right)$ в случае, если $v \in\left(X^{(n)}\left(t_{1}\right) \wedge X^{(n)}\left(t_{2}\right), X^{(n)}\left(t_{1}\right) \vee X^{(n)}\left(t_{2}\right)\right)$, и равен нулю в cлучае, когда $v \in \mathbf{R} \backslash\left[X^{(n)}\left(t_{1}\right) \wedge X^{(n)}\left(t_{2}\right), X^{(n)}\left(t_{1}\right) \vee X^{(n)}\left(t_{2}\right)\right]$.

Д оказ атель ств о. Заметим, что

$$
\int_{t_{1}}^{t_{2}} \operatorname{sign}\left(\left(X^{(n)}\right)^{\prime}(s)\right) N^{(n)}(d s, v)
$$


- это сумма знаков производной ломаной, взятых в точках пересечения ломаной $X^{(n)}(s)$ уровня $v$. Но с ростом переменной $s$ знаки производной ломаной чередуются, а число пересечений уровня $v$ ломаной $X^{(n)}(s)$ нечетно, если $v \in\left(X^{(n)}\left(t_{1}\right) \wedge X^{(n)}\left(t_{2}\right), X^{(n)}\left(t_{1}\right) \vee X^{(n)}\left(t_{2}\right)\right)$, и четно в случае, когда $v \in \mathbf{R} \backslash\left[X^{(n)}\left(t_{1}\right) \wedge X^{(n)}\left(t_{2}\right), X^{(n)}\left(t_{1}\right) \vee X^{(n)}\left(t_{2}\right)\right]$, откуда вытекает утверждение леммы 1 .

Теорема 1. Пусть функиии $X(s)$ u $f(s, u)$ удовлетворяют условию (S) на $[0, t]$, тогда справедливы следуюшие утверждения.

1. Симметричный интеграл

$$
\int_{0}^{t} f(s, X(s)) * d X(s)
$$

существует и вьчисляется по формуле:

$$
\begin{aligned}
\int_{0}^{t} f(s, X(s)) * d X(s)=-\int_{X(t)}^{X(0)} f\left(m^{-1}(v), v\right) d v \mathbf{1}(X(t)<X(0)) \\
\quad-\int_{X(t)}^{X(0)} \int_{m^{-1}(v)}^{t} \mathbf{1}(X(\tau)>v) f(d \tau, v) d v \mathbf{1}(X(t)<X(0)) \\
+\int_{m(t)}^{X(0)} \int_{m^{-1}(v)}^{t} \mathbf{1}(X(\tau)<v) f(d \tau, v) d v \mathbf{1}(X(t) \geqslant X(0)) \\
+\int_{m(t)}^{X(t)} \int_{m^{-1}(v)}^{t} \mathbf{1}(X(\tau)<v) f(d \tau, v) d v \mathbf{1}(X(t)<X(0)) \\
+\int_{X(0)}^{X(t)} f_{\left.M^{-1}(v), v\right) d v \mathbf{1}(X(t)>X(0))} \\
+\int_{X(0)}^{X(t)} \int_{M^{-1}(v)}^{t} \mathbf{1}(X(\tau)<v) f(d \tau, v) d v \mathbf{1}(X(t)>X(0)) \\
-\int_{X(0)}^{M(t)} \int_{M^{-1}(v)}^{t} \mathbf{1}(X(\tau)>v) f(d \tau, v) d v \mathbf{1}(X(t) \leqslant X(0)) \\
-\int_{X(t)}^{M(t)} \int_{M^{-1}(v)}^{t} \mathbf{1}(X(\tau)>v) f(d \tau, v) d v \mathbf{1}(X(t)>X(0))
\end{aligned}
$$

2. Если парь функиий $X(s), f_{1}(s, u)$ и $X(s), f_{2}(s, u)$ удовлетворяют условию (S) на $[0, t] u$

$$
f_{1}(s, X(s))=f_{2}(s, X(s)), \quad s \in[0, t]
$$

mo

$$
\int_{0}^{t} f_{1}(s, X(s)) * d X(s)=\int_{0}^{t} f_{2}(s, X(s)) * d X(s) .
$$

Д о к а з а т е л ь с т в о. 1 . Обозначим через $S_{t}^{(n)}(f, X)$ интегральную сумму, соответствующую симметричному интегралу $\int_{0}^{t} f(s, X(s))$ * 
$d X(s)$. Тогда, в силу существования локального времени (см. [10]) у ломаной $X^{(n)}(s)$, величина $S_{t}^{(n)}(f, X)$ равна

$$
\begin{gathered}
\int_{0}^{t} f\left(s, X^{(n)}(s)\right)\left(X^{(n)}\right)^{\prime}(s) d s=\left(\int_{m(t)}^{X(0)}+\int_{X(0)}^{M(t)}\right) \int_{0}^{t} f(s, v) \\
\times \operatorname{sign}\left(\left(X^{(n)}\right)^{\prime}(s)\right) N^{(n)}(d s, v) d v=J_{1}^{(n)}+J_{2}^{(n)}
\end{gathered}
$$

где $N^{(n)}(s, v)$ - индикатриса Банаха функции $X^{(n)}(\tau)$ на отрезке $[0, s]$.

Ниже анализируется слагаемое $J_{1}^{(n)}$; выражение $J_{2}^{(n)}$ исследуется аналогично. Мы выделим случай, когда $X(t) \neq X(0)$, на случай $X(t)=X(0)$ рассуждения переносятся тривиальным образом.

Заметим, что так как

$$
f(s, v)=f\left(m^{-1}(v), v\right)+\int_{m^{-1}(v)}^{s} f(d \tau, v), \quad \text { если } \quad m^{-1}(v) \leqslant s \leqslant t,
$$

и

$$
f(s, v)=f\left(m^{-1}(v), v\right)-\int_{s}^{m^{-1}(v)} f(d \tau, v), \quad \text { если } \quad 0 \leqslant s \leqslant m^{-1}(v),
$$

To

$$
\begin{aligned}
J_{1}^{(n)}= & \int_{m(t)}^{X(0)} f\left(m^{-1}(v), v\right) \int_{0}^{t} \operatorname{sign}\left(\left(X^{(n)}\right)^{\prime}(s)\right) N^{(n)}(d s, v) d v \\
& +\int_{m(t)}^{X(0)} \int_{m^{-1}(v)}^{t} \int_{m^{-1}(v)}^{s} f(d \tau, v) \operatorname{sign}\left(\left(X^{(n)}\right)^{\prime}(s)\right) N^{(n)}(d s, v) d v \\
& -\int_{m(t)}^{X(0)} \int_{0}^{m^{-1}(v)} \int_{s}^{m^{-1}(v)} f(d \tau, v) \operatorname{sign}\left(\left(X^{(n)}\right)^{\prime}(s)\right) N^{(n)}(d s, v) d v .
\end{aligned}
$$

Заметим, что множество $\left\{v: \int_{0}^{t} 1\left(X^{(n)}(s)=v\right) d s>0\right\}$ конечно или пусто, поэтому в дальнейшем мы будем им пренебрегать. Первое слагаемое в правой части в силу леммы 1 равно

$$
\begin{aligned}
& \int_{m(t)}^{X(0)} f\left(m^{-1}(v), v\right) \kappa\left(v, X(0), X^{(n)}(t)\right) d v \\
& =-\int_{X^{(n)}(t)}^{X(0)} f\left(m^{-1}(v), v\right) d v \mathbf{1}\left(X^{(n)}(t)<X(0)\right)=J_{11}^{(n)} .
\end{aligned}
$$

В оставшихся двух слагаемых поменяем местами внутренний и средний интегралы и с помощью леммы 1 вычислим внутренние интегралы:

$$
\begin{aligned}
\int_{m(t)}^{X(0)} & \int_{m^{-1}(v)}^{t} \int_{\tau}^{t} \operatorname{sign}\left(\left(X^{(n)}\right)^{\prime}(s)\right) N^{(n)}(d s, v) f(d \tau, v) d v \\
& -\int_{m(t)}^{X(0)} \int_{0}^{m^{-1}(v)} \int_{0}^{\tau} \operatorname{sign}\left(\left(X^{(n)}\right)^{\prime}(s)\right) N^{(n)}(d s, v) f(d \tau, v) d v
\end{aligned}
$$




$$
\begin{aligned}
= & \int_{m(t)}^{X(0)} \int_{m^{-1}(v)}^{t} \kappa\left(v, X^{(n)}(\tau), X^{(n)}(t)\right) f(d \tau, v) d v \\
& -\int_{m(t)}^{X(0)} \int_{0}^{m^{-1}(v)} \kappa\left(v, X(0), X^{(n)}(\tau)\right) f(d \tau, v) d v
\end{aligned}
$$

Обозначим последние два слагаемых через $J_{12}^{(n)}$ и $J_{13}^{(n)}$ соответственно. Обратимся к выражению $J_{12}^{(n)}$. Заметим, что внутренний интеграл в этом выражении может быть отличен от нуля только в том случае, если $X^{(n)}(t) \wedge X^{(n)}(\tau)<X(0)$. При этом возможны следующие случаи.

(1) Пусть $X^{(n)}(t)<X^{(n)}(\tau)$, тогда

$$
\begin{aligned}
J_{12}^{(n)} & =-\int_{m(t)}^{X(0)} \int_{m^{-1}(v)}^{t} \mathbf{1}\left(X^{(n)}(t)<v<X^{(n)}(\tau)\right) f(d \tau, v) d v \\
& =-\int_{X^{(n)}(t)}^{X(0)} \int_{m^{-1}(v)}^{t} \mathbf{1}\left(X^{(n)}(\tau)>v\right) f(d \tau, v) d v \mathbf{1}\left(X^{(n)}(t)<X(0)\right) .
\end{aligned}
$$

(2) Пусть $X^{(n)}(t)>X^{(n)}(\tau)$, тогда

$$
\begin{aligned}
J_{12}^{(n)}= & \int_{m(t)}^{X(0)} \int_{m^{-1}(v)}^{t} \mathbf{1}\left(X^{(n)}(\tau)<v<X^{(n)}(t)\right) f(d \tau, v) d v \\
= & \int_{m(t)}^{X(0)} \int_{m^{-1}(v)}^{t} \mathbf{1}\left(X^{(n)}(\tau)<v\right) f(d \tau, v) d v \mathbf{1}\left(X^{(n)}(t) \geqslant X(0)\right) \\
& +\int_{m(t)}^{X^{(n)}(t)} \int_{m^{-1}(v)}^{t} \mathbf{1}\left(X^{(n)}(\tau)<v\right) f(d \tau, v) d v \mathbf{1}\left(X^{(n)}(t)<X(0)\right) .
\end{aligned}
$$

Осталось рассмотреть выражение $J_{13}^{(n)}$; внутренний интеграл в этом выражении отличен от нуля, только если $X^{(n)}(\tau)<X(0)$. Следовательно,

$$
J_{13}^{(n)}=\int_{m(t)}^{X(0)} \int_{0}^{m^{-1}(v)} \mathbf{1}\left(X^{(n)}(\tau)<v\right) f(d \tau, v) d v .
$$

Но если $\tau<m^{-1}(v)$, то $X(\tau) \geqslant v$, и, значит,

$$
J_{13}^{(n)}=\int_{m(t)}^{X(0)} \int_{0}^{m^{-1}(v)} 1\left(X^{(n)}(\tau)<v<X(\tau)\right) f(d \tau, v) d v
$$

Остается перейти к пределу при $n \rightarrow \infty$ в полученных выше выражениях; для этого воспользуемся тем фактом, что в силу условия (S) при почти всех $v$ для любых $t_{1}, t_{2}, t_{1}<t_{2}$, справедливо соотношение

$$
\begin{aligned}
& \lim _{n \rightarrow \infty} \int_{t_{1}}^{t_{2}} \mathbf{1}\left(X^{(n)}(\tau)<v\right) f(d \tau, v) \\
& \quad=\lim _{n \rightarrow \infty} \int_{t_{1}}^{t_{2}} \mathbf{1}\left(X^{(n)}(\tau)<v, X(\tau) \neq v\right) f(d \tau, v) \\
& \quad=\int_{t_{1}}^{t_{2}} \mathbf{1}(X(\tau)<v) f(d \tau, v) .
\end{aligned}
$$


В силу полученных выше формул после предельного перехода имеем:

$$
\begin{aligned}
\lim _{n \rightarrow \infty} J_{1}^{(n)}= & -\int_{X(t)}^{X(0)} f\left(m^{-1}(v), v\right) d v \mathbf{1}(X(t)<X(0)) \\
& -\int_{X(t)}^{X(0)} \int_{m^{-1}(v)}^{t} \mathbf{1}(X(\tau)>v) f(d \tau, v) d v \mathbf{1}(X(t)<X(0)) \\
& +\int_{m(t)}^{X(0)} \int_{m^{-1}(v)}^{t} \mathbf{1}(X(\tau)<v) f(d \tau, v) d v \mathbf{1}(X(t) \geqslant X(0)) \\
& +\int_{m(t)}^{X(t)} \int_{m^{-1}(v)}^{t} 1(X(\tau)<v) f(d \tau, v) d v \mathbf{1}(X(t)<X(0)) .
\end{aligned}
$$

Для выражения $J_{2}^{(n)}$ из формулы (2.2) справедливы с очевидными изменениями аналогичные рассуждения.

2. Заметим, что ввиду непрерывности функции $X(s)$ при каждом $v$ множество $\{\tau: X(\tau)<v\}$ можно представить в виде не более чем счетного объединения непересекающихся интервалов $\left(s_{v}^{(k)}, t_{v}^{(k)}\right)$, причем точки $s_{v}^{(k)}$ и $t_{v}^{(k)}$ лежат в множестве уровня $M_{v}=\{s: X(s)=v\}$. Аналогичное замечание справедливо и для множества $\{\tau: X(\tau)>v\}$.

С другой стороны, из равенства $f_{1}(s, X(s))=f_{2}(s, X(s)), s \in[0,1]$, вытекает, что при каждом $v$ справедливо равенство $f_{1}(s, v)=f_{2}(s, v)$, $s \in M_{v}$, поэтому

$$
f_{1}\left(m^{-1}(v), v\right)=f_{2}\left(m^{-1}(v), v\right) \quad \text { для всех } \quad v \in[m(1), X(0)]
$$

И

$$
f_{1}\left(M^{-1}(v), v\right)=f_{2}\left(M^{-1}(v), v\right) \quad \text { для всех } \quad v \in[X(0), M(1)]
$$

Далее, ввиду приведенных выше соображений при условии, что $X(t)>v>m(t)$, имеем:

$$
\begin{aligned}
& \int_{m^{-1}(v)}^{t} \mathbf{1}(X(\tau)<v) f_{1}(d \tau, v)=\sum_{k}\left[f_{1}\left(t_{v}^{(k)}, v\right)-f_{1}\left(s_{v}^{(k)}, v\right)\right] \\
& =\sum_{k}\left[f_{2}\left(t_{v}^{(k)}, v\right)-f_{2}\left(s_{v}^{(k)}, v\right)\right]=\int_{m^{-1}(v)}^{t} 1(X(\tau)<v) f_{2}(d \tau, v) .
\end{aligned}
$$

Аналогичные соображения справедливы и для интегралов

$$
\int_{M^{-1}(v)}^{t} 1(X(\tau)>v) f_{1}(d \tau, v)
$$

при условии, что $X(t)<v<M(t)$. Поскольку все внутренние интегралы в формуле (2.1) имеют подобную структуру, то справедливо второе утверждение теоремы 1. 
3. Симметричные интегралы и потраекторные аналоги стохастических дифференциальных уравнений. Всюду в этом пункте, если не оговорено противное, фиксируется непрерывная функция $X(s), s \in[0,+\infty)$. Приведем другие способы вычисления симметричного интеграла.

Теорема 2. Пусть функиии $X(s), s \in[0, t], u f(s, u), s \in[0, t]$, $u \in \mathbf{R}$, удовлетворяют условию $(\mathrm{S})$ на $[0, t]$. Справедливь следующие утверждения.

1. Симметричныци интеграл $\int_{0}^{t} f(s, X(s)) * d X(s)$ может былть вычислен по одной из формул:

$$
\begin{aligned}
\int_{0}^{t} f(s, X(s)) * d X(s)= & \int_{X(0)}^{X(t)} f(0, v) d v \\
& +\int_{m(t)}^{M(t)} \int_{0}^{t} \kappa(v, X(\tau), X(t)) f(d \tau, v) d v \\
\int_{0}^{t} f(s, X(s)) * d X(s)= & \int_{X(0)}^{X(t)} f(t, v) d v \\
& -\int_{m(t)}^{M(t)} \int_{0}^{t} \kappa(v, X(0), X(\tau)) f(d \tau, v) d v
\end{aligned}
$$

2. Пусть функция $X(s)$ обладает локальным временем $\alpha(t, u)$. Тогда симметричный интеграл $\int_{0}^{t} f(s, X(s)) * d X(s)$ можно вычислить по формуле

$$
\begin{gathered}
\int_{0}^{t} f(s, X(s)) * d X(s)=\int_{X(0)}^{X(t)} f(\gamma(\alpha(t, v), v), v) d v \\
-\int_{m(t)}^{M(t)} \int_{0}^{\gamma(\alpha(t, v), v)} \kappa(v, X(0), X(t \wedge \tau)) f(d \tau, v) d v
\end{gathered}
$$

əде $\gamma(x, u)=\inf \{s: \alpha(s, u)>x\}$.

Доказательств о. 1. Пусть для простоты $t=1$. Обозначим через $S^{(n)}(f, X)$ интегральную сумму, соответствуюшую симметричному интегралу $\int_{0}^{1} f(s, X(s)) * d X(s)$. Тогда в силу формулы $(1.1)$, примененной к ломаной $X^{(n)}(s)$ и ее локальному времени, получим

$$
S^{(n)}(f, X)=\int_{m(1)}^{M(1)} \int_{0}^{1} f(s, v) \operatorname{sign}\left(\left(X^{(n)}\right)^{\prime}(s)\right) N^{(n)}(d s, v) d v
$$

где $N^{(n)}(s, v)$ - индикатриса Банаха ломаной $X^{(n)}(\tau)$ на отрезке $[0, s]$. Так как

$$
f(s, v)=f(0, v)+\int_{0}^{1} \mathbf{1}(\tau \leqslant s) f(d \tau, v)
$$


то внутренний интеграл в правой части равенства (3.4) можно записать в виде

$$
\begin{aligned}
& \int_{0}^{1} \int_{\tau}^{1} \operatorname{sign}\left(\left(X^{(n)}\right)^{\prime}(s)\right) N^{(n)}(d s, v) f(d \tau, v) \\
& \quad+f(0, v) \int_{0}^{1} \operatorname{sign}\left(\left(X^{(n)}\right)^{\prime}(s)\right) N^{(n)}(d s, v) .
\end{aligned}
$$

В силу леммы 1

$$
\int_{\tau}^{1} \operatorname{sign}\left(\left(X^{(n)}\right)^{\prime}(s)\right) N^{(n)}(d s, v)=\kappa\left(v, X^{(n)}(\tau), X(1)\right), \quad \tau \geqslant 0
$$

следовательно, интегральная сумма $S^{(n)}(f, X)$ равна

$$
\begin{array}{r}
\int_{m^{(n)}(1)}^{M^{(n)}(1)} \int_{0}^{1} \kappa\left(v, X^{(n)}(\tau), X(1)\right) f(d \tau, v) d v \\
+\int_{m^{(n)}(1)}^{M^{(n)}(1)} \kappa(v, X(0), X(1)) f(0, v) d v
\end{array}
$$

где

$$
m^{(n)}(1)=\min \left\{X^{(n)}(\tau): \tau \in[0,1]\right\}, \quad M^{(n)}(1)=\max \left\{X^{(n)}(\tau): \tau \in[0,1]\right\}
$$

Второе слагаемое в $(3.5)$ равно $\int_{X(0)}^{X(1)} f(0, v) d v$. Обратимся к первому слагаемому. Заметим, что внутренний интеграл в этом слагаемом в силу п. (b) условия (S) равен сумме интегралов

$$
\begin{aligned}
& \mathbf{1}(X(1) \geqslant v) \int_{0}^{1} \mathbf{1}\left(X^{(n)}(\tau)<v\right) f(d \tau, v) \\
& \quad+\mathbf{1}(X(1)<v) \int_{0}^{1} \mathbf{1}\left(X^{(n)}(\tau)>v\right) f(d \tau, v) .
\end{aligned}
$$

Так как последовательность ломаных $X^{(n)}(\tau)$ сходится к непрерывной функции $X(\tau)$, то в силу условия $(\mathrm{S})$ последнее выражение при почти всех $v$ стремится при $n \rightarrow \infty$ к выражению

$\mathbf{1}(X(1) \geqslant v) \int_{0}^{1} \mathbf{1}(X(\tau)<v) f(d \tau, v)+\mathbf{1}(X(1)<v) \int_{0}^{1} \mathbf{1}(X(\tau)>v) f(d \tau, v)$

следовательно, ввиду теоремы Лебега о предельном переходе под знаком интеграла, формула (3.1) доказана.

Доказательство формулы (3.2) аналогично с той лишь разницей, что оно опирается на соотношение $f(s, v)=f(t, v)-\int_{s}^{t} f(d \tau, v)$, остальные рассуждения аналогичны приведенным выше. 
2. Заметим, что $\gamma(\alpha(t, v), v) \geqslant t$, поэтому

$$
f(s, v)=f(\gamma(\alpha(t, v), v), v)-\int_{s}^{\gamma(\alpha(t, v), v)} f(d \tau, v) .
$$

Обозначим, как и выше, через $S_{t}^{(n)}(f, X)$ интегральную сумму, соответствующую симметричному интегралу $\int_{0}^{t} f(s, X(s)) * d X(s)$. Проведя такие же, как и выше, рассуждения, приходим к равенству

$$
\begin{aligned}
S_{t}^{(n)}(f, X)= & \int_{X^{(n)}(0)}^{X^{(n)}(t)} f(\gamma(\alpha(t, v), v), v) d v \\
& -\int_{m(t)}^{M(t)} \int_{0}^{t} \int_{s}^{\gamma(\alpha(t, v), v)} f(d \tau, v) \operatorname{sign}\left(\left(X^{(n)}\right)^{\prime}(s)\right) N^{(n)}(d s, v) d v
\end{aligned}
$$

Второе слагаемое ввиду теоремы Фубини преобразуется следуюшим образом:

$$
\begin{gathered}
\int_{m(t)}^{M(t)} \int_{0}^{\gamma(\alpha(t, v), v)} \int_{0}^{t} 1(s<\tau) \operatorname{sign}\left(\left(X^{(n)}\right)^{\prime}(s)\right) N^{(n)}(d s, v) f(d \tau, v) d v \\
=\int_{m(t)}^{M(t)} \int_{0}^{\gamma(\alpha(t, v), v)} \kappa\left(v, X(0), X^{(n)}(t \wedge \tau)\right) f(d \tau, v) d v .
\end{gathered}
$$

Дальнейшие рассуждения повторяют рассуждения, приведенные выше.

3 а м е ч а н и е. Пусть функция $f(s, u), s \in[0,1], u \in \mathbf{R}$, имеет непрерывную частную производную $\frac{\partial^{2}}{\partial s \partial u} f(s, u)$. Предположим, что функции $X(s)$ и $\frac{\partial}{\partial u} f(s, u)$ удовлетворяют условию $(\mathrm{S})$ на $[0,1]$. Тогда справедливо равенство

$$
\begin{aligned}
f(t, X(t))-f(0, X(0))= & \int_{0}^{t} \frac{\partial}{\partial u} f(s, X(s)) * d X(s) \\
& +\int_{0}^{t} \frac{\partial}{\partial s} f(s, X(s)) d s, \quad t \in[0,1] .
\end{aligned}
$$

Действительно, в силу формулы (3.2) имеем:

$$
\begin{aligned}
f(t, & X(t))-f(0, X(0))=\int_{X(0)}^{X(t)} \frac{\partial}{\partial u} f(t, u) d u+\int_{0}^{t} \frac{\partial}{\partial s} f(s, X(0)) d s \\
= & \int_{X(0)}^{X(t)} \frac{\partial}{\partial u} f(s, u) d u-\int_{0}^{t} \int_{X(0)}^{X(s)} \frac{\partial^{2}}{\partial s \partial u} f(s, u) d u d s \\
& +\int_{0}^{t}\left[\frac{\partial}{\partial s} f(s, X(0))+\int_{X(0)}^{X(s)} \frac{\partial^{2}}{\partial s \partial u} f(s, u) d u\right] d s \\
= & \int_{0}^{t} \frac{\partial}{\partial u} f(s, X(s)) * d X(s) \\
& +\int_{0}^{t}\left[\frac{\partial}{\partial s} f(s, X(0))+\frac{\partial}{\partial s} f(s, X(s))-\frac{\partial}{\partial s} f(s, X(0))\right] d s .
\end{aligned}
$$


Из соотношения (3.6), в частности, следует, что в случае, когда $X(s)=X(s, \omega)$ - стандартный винеровский процесс, а детерминированная функция $h(s, u)$ имеет непрерывную частную производную $\frac{\partial}{\partial u} h(s, u)$, формулу Ито можно записать в виде

$$
\begin{aligned}
\int_{0}^{t} h(s, X(s)) * d X(s)= & \int_{0}^{t} h(s, X(s)) d X(s) \\
& +\frac{1}{2} \int_{0}^{t} \frac{\partial}{\partial u} h(s, X(s)) d s
\end{aligned}
$$

где первое слагаемое в правой части есть стохастический интеграл Ито.

Рассмотрим уравнение

$$
\eta(t)-\eta(0)=\int_{0}^{t} a(s, \eta(s)) * d X(s)+\int_{0}^{t} b(s, \eta(s)) d s
$$

где первый интеграл есть симметричный интеграл по непрерывной функции $X(s)$ неограниченной вариации.

Уравнения такого вида являются детерминированными аналогами стохастических дифференциальных уравнений. С точки зрения теории случайных процессов, техника потраекторных симметричных интегралов позволяет отказаться от стандартных предположений о предсказуемости интеграндов в уравнениях такого вида и рассматривать подобные уравнения для произвольных непрерывных с вероятностью 1 случайных функций $X(s)=X(s, \omega)$ и случайных коэффициентов $a(s, \eta)$ и $b(s, \eta)$.

Решением уравнения (3.8) будем называть любую функцию вида

$$
\eta(s)=\phi(s, X(s))
$$

для которой имеют смысл интегралы в правой части уравнения (3.8) и которая обращает это уравнение в тождество.

В дальнейшем предполагается, если не оговорено противное, что все рассматриваемые ниже функиии имеют столько, сколько необходимо, непрерьвньх частных производньъ.

Покажем, что метод вычисления симметричного интеграла, приведенный в теореме 2, и формула (3.6) позволяют при определенных условиях гладкости коэффициентов уравнения свести решение уравнения (3.8) к решению системы обыкновенных дифференциальных уравнений первого порядка.

Действительно, предположим, что решение $\eta(s)=\phi(s, X(s))$ уравнения (3.8) с достаточно гладкой функцией $\phi(s, u)$ существует, и при этом предположении вычислим согласно формуле (3.6) симметричный интеграл в правой части уравнения (3.8):

$$
\int_{0}^{t} a(s, \phi(s, X(s))) * d X(s)=\int_{X(0)}^{X(t)} a(t, \phi(t, u)) d u
$$




$$
\begin{aligned}
& -\int_{0}^{t} \int_{X(0)}^{X(s)}[a(s, \phi(s, u))]_{s}^{\prime} d u d s=\int_{X(0)}^{X(t)} a(t, \phi(t, u)) d u \\
& -\int_{0}^{t} \int_{X(0)}^{X(s)}\left[a_{s}^{\prime}(s, \phi(s, u))+a_{\phi}^{\prime}(s, \phi(s, u)) \phi_{s}^{\prime}(s, u)\right] d u d s .
\end{aligned}
$$

Левую часть уравнения (3.8) мы можем записать в виде

$$
\begin{aligned}
\eta(t)-\eta(0) & =[\phi(t, X(t))-\phi(t, X(0))]+[\phi(t, X(0))-\phi(0, X(0))] \\
& =\int_{X(0)}^{X(t)} \phi_{u}^{\prime}(t, u) d u+\int_{0}^{t} \phi_{s}^{\prime}(s, X(0)) d s .
\end{aligned}
$$

Подставив соотношения (3.9) и (3.10) в уравнение (3.8), получим

$$
\begin{gathered}
\int_{X(0)}^{X(t)} \phi_{u}^{\prime}(t, u) d u+\int_{0}^{t} \phi_{s}^{\prime}(s, X(0)) d s=\int_{X(0)}^{X(t)} a(t, \phi(t, u)) d u \\
-\int_{0}^{t} \int_{X(0)}^{X(s)}[a(s, \phi(s, u))]_{s}^{\prime} d u d s+\int_{0}^{t} b(s, \eta(s)) d s,
\end{gathered}
$$

или

$$
\begin{aligned}
\int_{X(0)}^{X(t)}\left[\phi_{u}^{\prime}(t, u)-a(t, \phi(t, u))\right] d u & \\
=\int_{0}^{t}\{b(s, \phi(s, X(s))) & \\
& \left.\quad-\int_{X(0)}^{X(s)}[a(s, \phi(s, u))]_{s}^{\prime} d u-\phi_{s}^{\prime}(s, X(0))\right\} d s .
\end{aligned}
$$

Заметим, что правая часть равенства (3.11) является функцией ограниченной вариации, в то время как левая нет. Следовательно, мы можем приравнять интегранды в обеих частях равенства (3.11) к нулю. Мы получим систему

$$
\left\{\begin{array}{l}
\phi_{u}^{\prime}(s, u)=a(s, \phi(s, u)), \\
\phi_{s}^{\prime}(s, X(0))=b(s, \phi(s, X(s)))-\int_{X(0)}^{X(s)}[a(s, \phi(s, u))]_{s}^{\prime} d u .
\end{array}\right.
$$

Нам также необходимо начальное условие $\phi(0, X(0))=\eta(0)$.

Заметим, что, воспользовавшись первым уравнением системы, второе слагаемое из правой части второго уравнения можно преобразовать следующим образом:

$$
\int_{X(0)}^{X(s)}[a(s, \phi(s, u))]_{s}^{\prime} d u=\int_{X(0)}^{X(s)} \phi_{s u}^{\prime \prime}(s, u) d u=\phi_{s}^{\prime}(s, X(s))-\phi_{s}^{\prime}(s, X(0)) .
$$

Следовательно, система уравнений примет вид:

$$
\left\{\begin{array}{l}
\phi_{u}^{\prime}(s, u)=a(s, \phi(s, u)), \\
\phi_{s}^{\prime}(s, X(s))=b(s, \phi(s, X(s))), \quad \phi(0, X(0))=\eta(0) .
\end{array}\right.
$$


Таким образом, мы доказали следующий результат.

Теорема 3. Предположим, что непрерьвная функиия $X(s)$ на любом отрезке $[0, t]$ имеет неограниченную вариацию, а функиия $a(s, \phi)$ имеет непрерывнье частные производные первого порядка. Пусть функция $\phi=\phi(s, u)$ есть решение системы уравнений (3.12), тогда функиия $\eta(s)=\phi(s, X(s))$ является решением уравнения (3.8). Обратно, если существует гладкое решение уравнения (3.8) вида $\eta(s)=\phi(s, X(s))$, то функиия $\phi=\phi(s, u)$ удовлетворяет системе уравнений (3.12).

3 а м е ч а н и е. В случае негладких решений $\eta(s)=\phi(s, X(s))$ уравнения (3.8) с помощью формулы (3.2) и соотношений (3.9) и (3.10) можно вывести интегральный вариант системы уравнений (3.12).

Далее, решая первое уравнение системы в предположении, что решение сушествует, получим

$$
\int \frac{d \phi}{a(s, \phi)}=u+C(s)
$$

где $C(s)$ - произвольная постоянная, зависящая от переменной $s$. Заметим, что решение (3.13) первого уравнения системы определяет неявную функцию $\phi=\phi(s, u)$, которую можно также записать в виде $\phi=\phi^{*}(s, C(s), u)$.

Чтобы найти неизвестную функцию $C(s)$, воспользуемся вторым уравнением системы (3.12). Для этого при каждом фиксированном $u \in \mathbf{R}$ положим

$$
\Phi(s, \phi)=\Phi(s, \phi, u)=\int \frac{d \phi}{a(s, \phi)}-u-C(s)=0 .
$$

По теореме о производной неявной функции имеем:

$$
\begin{gathered}
\phi_{s}^{\prime}(s, u)=-\frac{\Phi_{s}^{\prime}(s, \phi, u)}{\Phi_{\phi}^{\prime}(s, \phi, u)}, \quad \Phi_{s}^{\prime}(s, \phi, u)=\left(\int \frac{d \phi}{a(s, \phi)}\right)_{s}^{\prime}-C_{s}^{\prime}(s), \\
\Phi_{\phi}^{\prime}(s, \phi, \phi)=\frac{1}{a(s, \phi)}
\end{gathered}
$$

следовательно,

$$
\phi_{s}^{\prime}(s, u)=a(s, \phi)\left[C_{s}^{\prime}(s)-\left(\int \frac{d \phi}{a(s, \phi)}\right)_{s}^{\prime}\right] .
$$

Подставляя найденную производную во второе уравнение системы, получим

$$
\begin{gathered}
C^{\prime}(s)=\frac{b\left(s, \phi^{*}(s, C(s), X(s))\right)}{a\left(s, \phi^{*}(s, C(s), X(s))\right)}-\left.\left(\int \frac{d \phi}{a(s, \phi)}\right)_{s}^{\prime}\right|_{\phi=\phi^{*}(s, C(s), X(s))}, \\
\phi^{*}(0, C(0), X(0))=\eta(0) .
\end{gathered}
$$


Предложенный выше метод может быть применим и к другим классам уравнений.

1. Рассмотрим уравнение

$$
\eta(t)-\eta(0)=\int_{0}^{t} a(s, X(s), \eta(s)) * d X(s)+\int_{0}^{t} b(s, X(s), \eta(s)) d s
$$

которое может быть решено аналогичным образом, при этом мы получаем систему уравнений

$$
\left\{\begin{array}{l}
\phi_{u}^{\prime}(s, u)=a(s, u, \phi(s, u)), \\
\phi_{s}^{\prime}(s, X(s))=b(s, X(s), \phi(s, X(s))) .
\end{array}\right.
$$

Первое уравнение системы уже не является уравнением с разделяющимися переменными, но его решение $\phi=\phi^{*}(s, C(s), u)$, если оно существует, содержит неизвестную функцию $C(s)$, которую можно найти с помощью второго уравнения системы (3.16) и начального условия $\phi^{*}(0, C(0), X(0))=\eta(0)$.

2. Рассмотрим уравнение вида

$$
\begin{aligned}
\eta(t)-\eta(0)= & \int_{0}^{t} a(s, X(s), \eta(s)-A(s)) * d X(s) \\
& +\int_{0}^{t} b(s, X(s), \eta(s)-A(s)) d s+A(t)-A(0),
\end{aligned}
$$

где $A(s)$ - известная функция. Заметим, что уравнение (3.17) эквивалентно уравнению (3.15) в случае, когда $A(s)$ - непрерывная справа функция ограниченной вариации, в противном случае это, вообще говоря, не так. Будем искать решение уравнения (3.17) в виде $\eta(s)=$ $\phi(s, X(s))+A(s)$, где $\phi(s, u)$ - достаточно гладкая функция. Тогда аналогичные рассуждения приводят к системе уравнений (3.16).

3. Напомним, следуя А.В. Скороходу (см. [8, с. 116-118]), что если задана непрерывная функция $\eta(s), s \in[0, T], \eta(0) \geqslant 0$, то пару функций $(Z(s), Y(s)), s \in[0, T]$, где $Y(s)=-\min \{\eta(\tau) \wedge 0: \tau \in[0, s]\}$, а $Z(s)=$ $\eta(s)+Y(s)$, называют решением задачи отражения для функции $\eta(s)$, а функцию $Z(s)$ - отраженной функцией.

Пусть $\eta(s)=\phi(s, X(s))$ есть решение уравнения (3.15). Положим $A(s)=-\min \{\eta(\tau) \wedge 0: \tau \in[0, s]\}$, тогда решение уравнения

$$
\begin{aligned}
\eta^{*}(t)-\eta(0)= & \int_{0}^{t} a\left(s, X(s), \eta^{*}(s)-A(s)\right) * d X(s) \\
& +\int_{0}^{t} b\left(s, X(s), \eta^{*}(s)-A(s)\right) d s+A(t)-A(0),
\end{aligned}
$$

где коэффициенты $a(s, y)$ и $b(s, y)$ такие же, как в уравнении $(3.15)$, доставляет нам «отраженную в нуле» функцию $Z(s)$. 
4. Пусть $X(s)=X(s, \omega), s \in[0,+\infty)$, - стандартный винеровский процесс. Рассмотрим стохастическое дифференциальное уравнение

$$
\eta(t)-\eta(0)=\int_{0}^{t} a(s, X(s), \eta(s)) d X(s)+\int_{0}^{t} b^{*}(s, X(s), \eta(s)) d s,
$$

где первый интеграл в правой части есть стохастический интеграл Ито. Предполагается, что коэффициенты $a(s, u, \phi)$ и $b^{*}(s, u, \phi)$ предсказуемы и удовлетворяют стандартным условиям существования соответствующих интегралов в правой части уравнения. Будем, следуя принятой идеологии, искать решение в виде $\eta(s)=\phi(s, X(s))$. Согласно формуле (3.7), имеем:

$$
\begin{array}{rl}
\int_{0}^{t} & a(s, X(s), \phi(s, X(s))) d X(s) \\
\quad= & \int_{0}^{t} a(s, X(s), \phi(s, X(s))) * d X(s)-\int_{0}^{t} a^{*}(s, X(s), \phi(s, X(s))) d s
\end{array}
$$

где

$$
a^{*}(s, u, \phi)=\frac{1}{2}\left[\frac{\partial}{\partial u} a(s, u, \phi)+\frac{\partial}{\partial \phi} a(s, u, \phi) \frac{\partial}{\partial u} \phi(s, u)\right] .
$$

Тогда уравнение (3.18) эквивалентно уравнению

$$
\eta(t)-\eta(0)=\int_{0}^{t} a(s, X(s), \eta(s)) * d X(s)+\int_{0}^{t} b(s, X(s), \eta(s)) d s,
$$

где

$$
b(s, X(s), \phi(s, X(s)))=b^{*}(s, X(s), \phi(s, X(s)))-a^{*}(s, X(s), \phi(s, X(s))) .
$$

Решение последнего уравнения сводится к решению системы уравнений (3.16). Заметим, что если воспользоваться первым уравнением этой системы, то

$$
b(s, u, \phi)=b^{*}(s, u, \phi)-\frac{1}{2} \frac{\partial}{\partial u} a(s, u, \phi)-\frac{1}{4} \frac{\partial}{\partial \phi} a^{2}(s, u, \phi) .
$$

Наконец, в силу соотношений (3.16) и (3.19) из формулы (3.20) следует, что решение $\phi=\phi(s, u)$ стохастического дифференциального уравнения (3.18) удовлетворяет равенству

$$
\frac{\partial}{\partial s} \phi(s, X(s))=b^{*}(s, X(s), \phi(s, X(s)))-\frac{1}{2} \frac{\partial^{2}}{\partial u^{2}} \phi(s, X(s)) .
$$

Для иллюстрации рассмотренных выше методов приведем следующий пример. 
П р и м е р. Бесселевские диффузии [1, с. 226-228]. Рассмотрим стохастическое дифференциальное уравнение

$$
\eta(t)-\eta(0)=2 \int_{0}^{t}(\eta(s) \vee 0)^{1 / 2} d X(s)+\alpha t, \quad \eta(0)=x^{2},
$$

где интеграл в правой части уравнения есть стохастический интеграл Ито по винеровскому процессу $X(s), X(0)=0$. Решение данного уравнения доставляет квадрат бесселевского процесса. Поскольку в силу преобразования (3.21) получим $b(s, X(s), \eta(s))=\alpha-\frac{\partial}{\partial \eta}(\eta(s) \vee 0)$, то система (3.16), отвечающая уравнению (3.22), имеет вид

$$
\left\{\begin{array}{l}
\phi_{u}^{\prime}(s, u)=2(\phi(s, u) \vee 0)^{1 / 2} \\
\phi_{s}^{\prime}(s, X(s))=\alpha-(\phi(s, u) \vee 0)_{\phi}^{\prime}, \quad \phi(0, X(0))=x^{2} .
\end{array}\right.
$$

Будем искать неотрицательные решения уравнения (3.22), в этом случае из первого уравнения системы выводим: $\int \phi^{-1 / 2} d \phi=2 u+2 C(s)$ или $\phi=(u+C(s))^{2}$. Следовательно, бесселевский процесс $\mathscr{B}(s)$ имеет такую структуру: $\mathscr{B}(s)=|X(s)+C(s)|$, где неизвестная гладкая функция $C(s)$ определяется из второго уравнения системы:

$$
C^{\prime}(s)=\frac{\alpha-1}{2(X(s)+C(s))}, \quad C^{2}(0)=x^{2} .
$$

В случае $\alpha=1, x=0$ имеем: $\phi(s, X(s))=X^{2}(s)$.

4. Расширенный симметричный интеграл и интеграл Хеллингера. Расширенные симметричные интегралы возникли (см. [6]) как несобственные симметричные интегралы в процессе исследования симметричных интегралов. Конструкция расширенного симметричного интеграла опирается на следующее утверждение.

Лемма 2. Пусть $X(s), s \in[0, t],-$ непрерывная функиия с локальным временем $\alpha(s, u)$, а $f(s), s \in[0, t],-$ суммируемая функиия. Тогда $f(s)=f_{t}^{+}(\xi(s), X(s))$ при почти всех $s \in[0, t]$, где $f_{t}^{+}(x, u)=$ $f(\gamma(x, u)) \mathbf{1}(\alpha(t, u) \geqslant x), \gamma(z, u)=\inf \{s: \alpha(s, u)>z\}, \xi(s)=\alpha(s, X(s))$.

О п р е д е лен и е. Функция $f_{t}^{+}(x, u)$, так же как и функция $f_{t}^{+}(\xi(s), X(s))$, называется представлением функиии $f(s)$ на отрезке $[0, t]$.

Расширенный симметричный интеграл строится для определенного класса интеграндов вида $f(\xi(s), X(s))$, и приведенная выше лемма 2 показывает, что класс интеграндов достаточно широк в том смысле, что для каждой суммируемой на $[0, t]$ функции $f(s)$ существует представление $f_{t}^{+}(\xi(s), X(s))$, для которого расширенный симметричный интеграл будет определен. 
О п р е д е л е н и е. Пусть $X(s), s \in[0,+\infty),-$ непрерывная функция с локальным временем $\alpha(t, u)$. Расширенным симметричным интегралом $(E) \int_{0}^{t} f(s) * d X(s)$ называется интеграл по заряду:

$$
\text { (E) } \begin{aligned}
\int_{0}^{t} f(s) * d X(s) & \equiv \int_{0}^{t} f_{t}^{+}(\xi(s), X(s)) * d X(s) \\
& =\int_{\mathbf{R}^{+} \times \mathbf{R}} f_{t}^{+}(x, u) G_{t}(d x d u),
\end{aligned}
$$

где $G_{t}(d x d u)$ - заряд, однозначно определяющийся своими значениями на «прямоугольниках» $A \times \mathscr{B}:$

$$
\begin{aligned}
G_{t}(A \times \mathscr{B}) \equiv & \int_{X(0)}^{X(t)} \mathbf{1}((\alpha(t, u), u) \in A \times \mathscr{B}) d u \\
& -\frac{1}{2} \int_{\mathbf{R}} \mathbf{1}((\alpha(t, u), u) \in(A \backslash\{0\}) \times \mathscr{B}) \operatorname{sign}(u-X(0)) d u \\
& +\frac{1}{2} \int_{\mathbf{R}} \mathbf{1}(u \in \mathscr{B}, \alpha(t, u)>0) \operatorname{sign}(u-X(0)) d u \mathbf{1}(0 \in A) .
\end{aligned}
$$

Расширенный симметричный интеграл может быть вычислен по формуле

$$
\begin{aligned}
& \int_{\mathbf{R}^{+} \times \mathbf{R}} f_{t}^{+}(x, u) G_{t}(d x d u)=\int_{X(0)}^{X(t)} f_{t}^{+}(\alpha(t, u), u) d u \\
& -\frac{1}{2} \int_{m(t)}^{M(t)}\left[f_{t}^{+}(\alpha(t, u), u)-f_{t}^{+}(0, u)\right] \operatorname{sign}(u-X(0)) d u .
\end{aligned}
$$

В работе [6] было показано, что заряд $G_{t}(d x d u)$, точнее, его значения на «прямоугольниках», могут быть получены как пределы симметричных интегралов с определенного вида интеграндами. Покажем, что для определенного класса интеграндов расширенный симметричный интеграл может быть получен как предел собственных симметричных интегралов, т.е. является несобственным симметричным интегралом.

Теорема 4. Пусть функиии $X(s), s \in[0,+\infty), u f(s, u), s \in$ $[0,+\infty), u \in \mathbf{R}$, удовлетворяют условию (S), u, кроме того, функиия $X(s)$ обладает локальным временем $\alpha(t, u)$. Обозначим $\gamma(x, v)=$ $\inf \{s: \alpha(s, v)>x\}, f_{\varepsilon}(s, u)=(1 /(2 \varepsilon)) \int_{u-\varepsilon}^{u+\varepsilon} f(\gamma(\alpha(s, v), v), v) d v$.

Тогда справедливо равенство

$$
\lim _{\varepsilon \downarrow 0} \int_{0}^{t} f_{\varepsilon}(s, X(s)) * d X(s)=(E) \int_{0}^{t} f(s, X(s)) * d X(s) .
$$

Д о к а з а т е л ь с т в о. Заметим, что функции $X(s)$ и $f_{\varepsilon}(s, u)$ удовлетворяют условию (S). Поэтому в силу соотношения

$$
f(\gamma(\alpha(s, v), v), v)=f(\gamma(\alpha(t, v), v), v)-\int_{\gamma(\alpha(s, v), v)}^{\gamma(\alpha(t, v), v)} f(d \tau, v), \quad s<t,
$$


имеем:

$$
\begin{gathered}
\int_{0}^{t} f_{\varepsilon}(s, X(s)) * d X(s) \\
=\lim _{n \rightarrow \infty} \int_{\mathbf{R}} \int_{0}^{t} \frac{1}{2 \varepsilon} \int_{u-\varepsilon}^{u+\varepsilon} f(\gamma(\alpha(s, v), v), v) d v \\
\times \operatorname{sign}\left(\left(X^{(n)}(s)\right)^{\prime}\right) N^{(n)}(d s, u) d u \\
=\lim _{n \rightarrow \infty} \int_{\mathbf{R}} \frac{1}{2 \varepsilon} \int_{u-\varepsilon}^{u+\varepsilon} f(\gamma(\alpha(t, v), v), v) d v \\
\quad \times \int_{0}^{t} \operatorname{sign}\left(\left(X^{(n)}(s)\right)^{\prime}\right) N^{(n)}(d s, u) d u \\
\quad \lim _{n \rightarrow \infty} \int_{\mathbf{R}} \frac{1}{2 \varepsilon} \int_{u-\varepsilon}^{u+\varepsilon}\left[\int_{0}^{t} \int_{\gamma(\alpha(s, v), v)}^{\gamma(\alpha(t, v), v)} f(d \tau, v)\right. \\
\left.\times \operatorname{sign}\left(\left(X^{(n)}(s)\right)^{\prime}\right) N^{(n)}(d s, u)\right] d v d u .
\end{gathered}
$$

Ввиду леммы 1 , учитывая, что если $s<\gamma(\alpha(\tau, v), v)$, то $\gamma(\alpha(s, v), v)<\tau$, мы можем представить выражение в квадратных скобках в последнем слагаемом в следующем виде:

$$
\begin{aligned}
& \int_{\gamma(0, v)}^{\gamma(\alpha(t, v), v)} \int_{0}^{t} \mathbf{1}(\gamma(\alpha(s, v), v)<\tau) \operatorname{sign}\left(\left(X^{(n)}(s)\right)^{\prime}\right) N^{(n)}(d s, u) f(d \tau, v) \\
& =\int_{\gamma(0, v)}^{\gamma(\alpha(t, v), v)} \kappa\left(u, X(0), X^{(n)}(\gamma(\alpha(s, v), v))\right) f(d \tau, v) .
\end{aligned}
$$

Переходя в этом выражении к пределу при $n \rightarrow \infty$, получим

$$
\int_{\gamma(0, v)}^{\gamma(\alpha(t, v), v)} \kappa(u, X(0), X(\gamma(\alpha(s, v), v))) f(d \tau, v) .
$$

Поскольку при почти всех $v$ справедливо равенство $X(\gamma(\alpha(s, v), v))=v$, то последний интеграл при почти всех $v$ равен

$$
\kappa(u, X(0), v)[f(\gamma(\alpha(t, v), v))-f(\gamma(0, v))]
$$

Следовательно, симметричный интеграл $\int_{0}^{t} f_{\varepsilon}(s, X(s)) * d X(s)$ равен

$$
\begin{aligned}
& \int_{X(0)}^{X(t)} f_{\varepsilon}(t, u) d u \\
& \quad-\int_{m(t)}^{M(t)} \frac{1}{2 \varepsilon} \int_{u-\varepsilon}^{u+\varepsilon} \kappa(u, X(0), v)[f(\gamma(\alpha(t, v), v))-f(\gamma(0, v))] d v d u .
\end{aligned}
$$

Переходя к пределу при $\varepsilon \downarrow 0$ в последнем выражении, получим формулу (4.2).

3 а м е ч а н и е. Пусть $X(s), s \in[0,+\infty)$, - непрерывная функция с локальным временем $\alpha(t, u)$, непрерывным по $t$ при почти 
всех $u$. Тогда для любой борелевской функции $f(t, u)$ вида $f(t, u)=$ $\int_{-\infty}^{u} g\left(\gamma^{*}(\alpha(t, v), v)\right) \mathbf{1}(\alpha(t, v)>0) d v$, где $g(t, v)$ при каждом $t$ является ограниченной функцией по переменной $u$, справедлива формула

$$
\begin{aligned}
& f(t, X(t))-f(0, X(0))=(E) \int_{0}^{t} \frac{\partial}{\partial u} f(s, X(s)) * d X(s) \\
& +\frac{1}{2}[f(t, M(t))+f(t, m(t))-f(0, M(t))-f(0, m(t))] .
\end{aligned}
$$

Заметим, что $f(t, X(t))-f(0, X(0))=[f(t, X(t))-f(t, X(0))]+$ $[f(t, X(0))-f(0, X(0))]$, в силу представления для функции $f(t, u)$ первое слагаемое равно

$$
\left.f(t, X(t))-f(t, X(0))=\int_{X(0)}^{X(t)} \frac{\partial}{\partial u} f(t, u)\right) d u
$$

а второе можно записать в виде

$$
\begin{aligned}
& f(t, X(0))-f(0, X(0))=\frac{1}{2} \int_{m(t)}^{M(t)}[f(t, u)-f(0, u)] 2 \delta(u-X(0)) d u \\
& \quad=\frac{1}{2} \int_{m(t)}^{M(t)}[f(t, u)-f(0, u)](\operatorname{sign}(u-X(0)))^{\prime} d u .
\end{aligned}
$$

Воспользовавшись формулой интегрирования по частям, правую часть последнего равенства представим в виде

$$
\begin{aligned}
(f(t, u) & -f(0, u))\left.\operatorname{sign}(u-X(0))\right|_{m(t)} ^{M(t)} \\
& -\frac{1}{2} \int_{m(t)}^{M(t)} \frac{\partial}{\partial u}[f(t, u)-f(0, u)] \operatorname{sign}(u-X(0)) d u \\
= & \frac{1}{2}[(f(t, M(t))-f(0, M(t))) \cdot 1-(f(t, m(t))-f(0, m(t))) \cdot(-1)] \\
& -\frac{1}{2} \int_{m(t)}^{M(t)}\left[g\left(\gamma^{*}(\alpha(t, u), u), u\right)-g(\gamma(0, u), u)\right] \operatorname{sign}(u-X(0)) d u
\end{aligned}
$$

откуда, согласно формуле для вычисления расширенного симметричного интеграла, получим искомое равенство.

Другой класс несобственных симметричных интегралов связан с интегралами Хеллингера. Напомним, что если $X(s), s \in[0,+\infty)$, - непрерывная функция, то интегралом Хеллингера называется

$$
(H) \int_{0}^{t} f(s) * d X(s)=\lim _{n \rightarrow \infty} \sum_{k} \frac{1}{\Delta t_{k}^{(n)}} \int_{\left[\Delta t_{k}^{(n)}\right]} f(s) d s \Delta X_{k}^{(n)} .
$$

Интегралы Хеллингера, как и симметричные интегралы, являются обобщениями интегралов Римана-Стилтьеса. Ряд сведений об условиях существования интегралов такого вида приведен в работах [2], [3]. 
В работе [6] была установлена связь между определенным классом несобственных симметричных интегралов и интегралами Хеллингера, ниже мы обобщим эти результаты. Пусть $X(s), Y(s), s \in[0, t],-$ непрерывные функции. Рассмотрим последовательность сгущающихся разбиений $\left\{t_{k}^{(n)}\right\}$ отрезка $[0, t]$ и обозначим через $Y^{(n)}(s), s \in[0, t]$, последовательность ломаных, построенных по функции $Y(s)$ и отвечающих этим разбиениям.

Теорема 5. Пусть функиии $X(s)$ u $f(s, u)$ удовлетворяют условию (S) на $[0, t]$, а $Y(s)$ - непрерьвная функиия. Если существует интеграл Хеллингера

$$
(H) \int_{0}^{t}\left(\int_{X(0)}^{X(s)} f(s, u) d u\right) d Y(s)
$$

то справедлива формула

$$
\begin{aligned}
(H) & \int_{0}^{t}\left(\int_{X(0)}^{X(s)} f(s, u) d u\right) d Y(s)=Y(t) \int_{X(0)}^{X(t)} f(t, u) d u \\
& -\int_{\mathbf{R}} \int_{0}^{t} \kappa(u, X(0), X(s)) Y(s) f(d s, u) d u \\
& -\lim _{n \rightarrow \infty} \int_{0}^{t} f(s, X(s)) Y^{(n)}(s) * d X(s) .
\end{aligned}
$$

Д о к а з а т е л ь с т в о. В силу формулы (3.2) имеем:

$$
\begin{aligned}
\int_{0}^{t} f(s, X(s)) Y^{(n)}(s) * d X(s)=Y^{(n)}(t) \int_{X(0)}^{X(t)} f(t, u) d u \\
\quad-\int_{\mathbf{R}} \int_{0}^{t} \kappa(u, X(0), X(s)) Y^{(n)}(s) f(d s, u) d u \\
-\int_{0}^{t} \int_{X(0)}^{X(s)} f(s, u) d u\left(Y^{(n)}\right)^{\prime}(s) d s .
\end{aligned}
$$

Последнее слагаемое в правой части соотношения (4.4) можно переписать как частичную сумму

$$
\sum_{k} \frac{1}{\Delta t_{k}^{(n)}} \int_{\left[\Delta t_{k}^{(n)}\right]}\left(\int_{X(0)}^{X(s)} f(s, u) d u\right) d s \Delta Y_{k}^{(n)}, \quad \Delta Y_{k}^{(n)}=Y\left(t_{k}^{(n)}\right)-Y\left(t_{k-1}^{(n)}\right),
$$

соответствующую интегралу

$$
(H) \int_{0}^{t}\left(\int_{X(0)}^{X(s)} f(s, u) d u\right) d Y(s) .
$$

Переходя к пределу при $n \rightarrow \infty$ в равенстве (4.4) и учитывая тот факт, что предел в левой части этого равенства существует в силу существования интеграла Хеллингера в правой части равенства, приходим к формуле (4.3). 


\section{СПИСОК ЛИТЕРАТУРЫ}

1. Ватанабэ С., Икэда Н. Стохастические дифференциальные уравнения и диффузионные процессы. М.: Наука, 1986, 445 с.

2. Дьячков А.М. О существовании интеграла Стилтьеса. - Докл. РАН, 1996, т. 350 , № 2 , c. $158-161$.

3. Мачаев В.И., Соломяк М. З. Об условиях существования интеграла Стилтьеса. - Матем. сб., 1972, т. 88, № 4, с. 522-535.

4. Натансон И. П. Теория функций вешественной переменной. М.: Наука, 1974, $480 \mathrm{c.}$

5. Насыров Ф. С. О локальных временах для функций и случайных процессов, I. Теория вероятн. и ее примен., 1995, т. 40, в. 4, с. 798-812.

6. Насыров Ф.С. Симметричные интегралы и их применение в финансовой математике. - Труды МИАН, 2002, т. 237, с. 265-278.

7. Терехин А. П. Приближение функций ограниченной $p$-вариации. - Изв. вузов, 1965 , № 2, c. 171-187.

8. Чжун К., Уильямс Р. Введение в стохастическое интегрирование. - М.: Мир, $1987,152 \mathrm{c}$.

9. Berman S. M. Nonincrease almost everywhere of certain measurable functions with applications to stochastic processes. - Proc. Amer. Math. Soc., 1983, v. 88, № 1, p. 141-144.

10. Geman D., Horowitz J. Occupation densities. - Ann. Probab., 1980, v. 8, № 1, p. 1-67.

11. Kondurar V. Sur l'intégrale de Stieltjes. - Матем. сб., 1937, т. 2, № 2, c. 361-366.

12. Young L. C. An inequality of the Hölder type, connected with Stieltjes integration. Acta Math., 1936, v. 67, p. 251-282.

Поступила в редакцию

23.IV.2003 